\title{
Age-dating Stellar Populations of Luminous Red Galaxies
}

\author{
Ando Ratsimbazafy ${ }^{1}$, Catherine Cress ${ }^{1}$, Steve Crawford ${ }^{2}$, \\ Claudia Maraston ${ }^{3}$, Robert $\mathrm{Nichol}^{3}$, and Daniel Thomas ${ }^{3}$ \\ ${ }^{1}$ Physics Department, University of the Western Cape, Private bag X17, \\ Cape Town 7535, South Africa \\ ${ }^{2}$ South African Astronomical Observatory, PO Box 9, Observatory 7935, \\ Cape Town, South Africa \\ ${ }^{3}$ Institute of Cosmology and Gravitation, University of Portsmouth, Dennis Sciama Building, \\ Burnaby road, Portsmouth PO1 3FX, The United Kingdom \\ email: raljha.a@gmail.com
}

\begin{abstract}
We investigate the possibility of using Luminous Red Galaxies (LRGs) as "Cosmic chronometers" to measure the expansion rate of the universe to $3 \%$ over a redshift range $0.1<$ $z<1.0$. In this method, $\mathrm{H}(\mathrm{z})$ is directly measured by using the ages of passively evolving galaxies to determine $d z / d t$. We first present results from our study of LRGs in simulations Crawford et al. (2010) where we explore the impact of extended star formation histories on the measurements of the Hubble parameter. We then extract a carefully selected sample of LRGs taken from Sloan Digital Sky Survey (SDSS) Data Released Seven (DR7), stack spectra in redshift bins to increase the signal-to-noise, and use the Lick index modelling presented in Thomas et al. (2011) to agedate the sample. We discuss the implications for expansion rate measurements and outline a proposal to observe LRGs with the Southern African Large Telescope (SALT).
\end{abstract}

Keywords. galaxies: evolution, cosmology: cosmological parameters , cosmology: observations

\section{Introduction}

The expansion rate of the Universe can potentially be measured by age-dating LRGs and using them as "Cosmic Chronometers" (CC). Most cosmological probes only measure the expansion rate integrated along a line-of-sight, however, the CC method allows a measurement at a specific redshift and this can provide tighter constraints on cosmological parameters (Jimenez \& Loeb 2002). In broad terms the basic premise is that stars in LRGs are formed at a similar time in the past, and by measuring the age difference between two ensembles of passively-evolving LRGs at different redshifts one can calculate the time interval, $\Delta t$, associated with a given redshift interval, $\Delta z$. The Hubble parameter within the redshift interval is then given by

$$
H(z)=-\frac{1}{1+z} \frac{\Delta z}{\Delta t}
$$

A number of scientists have attempted to use this method to track the evolution of $\mathrm{H}(\mathrm{z})$ as a function of redshift and place constraints on cosmological parameters (e.g. Jimenez et al. 2003, Simon et al. 2005, Carson \& Nichol 2010). For example in Jimenez et al. 2003, using SDSS data, they measured the Hubble constant of $H_{0}=69 \pm 12 \mathrm{~km} \mathrm{~s}^{-1} \mathrm{Mpc}^{-1}$. 


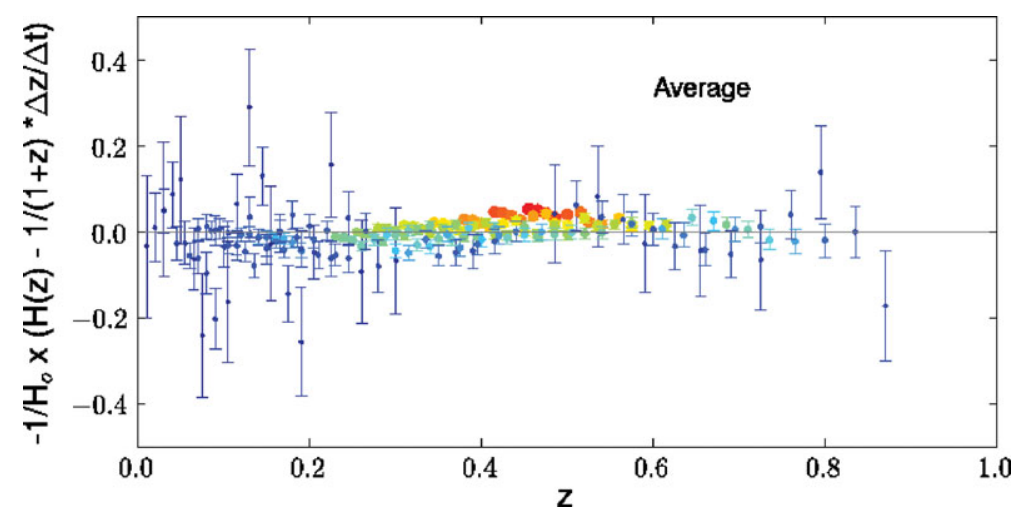

Figure 1. The calculated values of $\Delta z / \Delta t$ (data points) and the expected value for $\mathrm{H}(\mathrm{z})$ (solid line). $\Delta z / \Delta t$ were calculated from the age of simulated LRGs in MS. Each point represents the distance between the redshift gaps. The error in $\mathrm{H}(\mathrm{z})$ is associated with the inverse of difference in age hence the closer the redshift bins, the larger the error.

\section{LRGs in simulations}

In Crawford et al. (2010), we explored the validity of the assumptions implicit in the CC method using LRGs identified in the Millennium Simulation (MS). We used stellar population modelling and spectral synthesis to estimate the errors on ages that can be expected. Based on these we discussed optimization of such an experiment using SALT. Using our simulated set of galaxies, we found that $\mathrm{H}(\mathrm{z})$ can be recovered with a high accuracy of $<3 \%$ at $z \sim 0.4$ (Figure 1 ).

\section{High signal-to-noise LRG spectra}

We selected a homogeneous sample of LRGs from the SDSS catalogue in the redshift range of $0.31<z<0.55$. We then extracted galaxies which do not show either star formation or AGN-like activity. In order to achieve this, we measured the different emission lines in these galaxies and stellar kinematics using GANDALF (Gas AND Absorption Line Fitting) routine. We considered objects without $H \beta, H \alpha$ and [OIII] $\lambda \lambda 5007$ emission lines. In the fitting process to recover the stellar velocity (V) and the velocity dispersion $(\sigma)$, we used the Bruzual \& Charlot (2003) stellar population model used by Tremonti et al. (2004) combined with stellar templates based on MILES stellar library (Sánchez-Blázquez et al. 2006). We imposed all forbidden lines to have the kinematics of $[\mathrm{NII}] \lambda \lambda 6583$ and all recombination lines to have the kinematics of $H \alpha$. In addition, the $\mathrm{NaD}$ and skyline regions were excluded during the procedure.

To have accurate and precise derived SSP parameters, we combined the individual spectra into high signal-to-noise spectrum at each redshift bin. The effect of co-adding spectra before the line-strength measurement is that skyline residuals and the telluric absorption features have less impact. The line-strengths of the Lick indices were measured using the INDEXF program on co-added spectra. We included the contribution of the measured line-strength error from the uncertainty in the radial velocity. This is added in quadrature to the random error in the index line-strength arising from photon statistics and read-out noise.

The Lick index measurements were also corrected for kinematic broadening caused by the line-of-sight velocity distribution. We compared our measured Lick indices: $H \beta$, Mgb, Fe5270, Fe5335 to the predicted indices of Thomas et al. (2011). This calibrated 


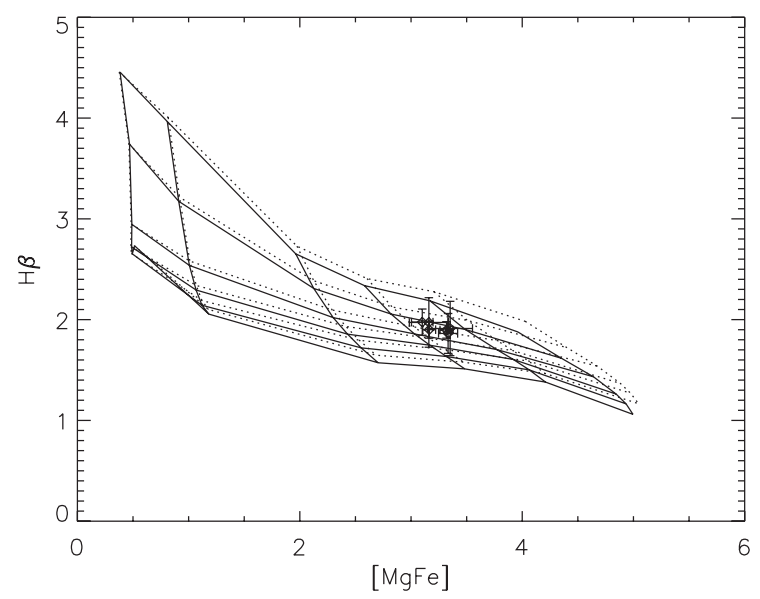

Figure 2. Index -index plots. Measured line strengths plotted over Thomas et al. (2011) model grids with $[\mathrm{E} / \mathrm{Fe}]=0$ (solid lines) and $[\mathrm{E} / \mathrm{Fe}]=0.3$ (dashed lines). From the bottom, age lines are $15,12,10,8,5,3$ Gyr. From right to left, $[\mathrm{Z} / \mathrm{H}]=0.67,0.35,0.00,-1.35,-2.25$. The composite index $[\mathrm{MgFe}]$ is calculated as defined in Thomas et al. (2011).

model makes use of the MILES stellar library, includes alpha element enhancements and is found to be comparable to the SDSS spectral resolution.

\section{Summary and future work}

We have stacked LRG spectra within redshift bins of $\Delta z=0.10$ in order to have high SNR spectra. We have measured Lick indices and compared them with the model of Thomas et al. (2011) to extract all stellar parameters. Our preliminary results show that ages vary from $8-11$ Gyr for LRGs in the range of $0.31<z<0.37$ (Figure 2). Age measurements up to $z=0.55$ are ongoing which will be combined with these to estimate the Hubble parameter.

An observation of LRGs at $z=0.40$ and $z=0.55$ with SALT as a pilot survey is underway. A minimum of $\mathrm{SNR}=10$ per resolution element with a rest frame resolution of $3 \AA$ will be obtained to age date the LRGs to $10 \%$ by means of matching the resolution of model SEDs. The use of BOSS data in 2012 is also a possibility.

\section{References}

Bruzual, G. \& Charlot, S. 2003, MNRAS, 344, 1000

Carson, D. \& Nichol, R. 2010, MNRAS, 408, 213

Crawford, S. M., Ratsimbazafy, A. L., Cress, C. M. et al. 2010, MNRAS, 406, 2569

Jimenez, R., Verde, L., Treu, T., \& Stern, D. 2003, ApJ, 593, 622

Jimenez, R. \& Loeb, A. 2002, ApJ, 573, 37

Sánchez-Blázquez P., Peletier, R. F., Jiménez-Vicente, J. et al. 2006, MNRAS, 371, 703

Simon, J., Verde, L., \& Jimenez, R. 2005, PhRvD, 71, 123001

Thomas, D., Maraston, C., \& Johansson, J. 2011, MNRAS, 412, 2183

Tremonti C. A., Heckman, T. M., Kauffmann, G. et al. 2004, ApJ, 613, 898 